\title{
Biobanking Education
}

\author{
Marcos Castellanos-Uribe, Emmanualle Gormally, ${ }^{2-4}$ Hongkang Zhou, \\ Elisabeth Matzke, ${ }^{6}$ and Peter $\mathrm{H}$. Watson ${ }^{6,7}$
}

B IOBANKING IS BECOMING more and more professional, which is why we have a specific need for further education and training. This need is not only for the staff working within and managing biobanks but also to train young graduates who are not familiar with the field but want to get involved in providing a service to biospecimen-based research.

We currently have a broad range of types of biobanks and employees with a wide variety of backgrounds - from doctoral students and technical staff in the research laboratory to the biobank professional with specialized expertise of a centralized biobank. The challenge is how to develop an appropriate range of education and training opportunities for the diverse needs of these people. We believe this will also contribute not only to the development of the biobanking field and recognition of its challenges by life and health scientists, but also to improve the knowledge of biobanking among other stakeholders such as the decision makers, general public, donors, and ethics committees.

Just over a decade ago, no formal biobank education programs for biobanking existed; typically, pathologists and scientists, self-educated about biobanking, directed activities related to research specimen collections and staff transferred knowledge from related or unrelated areas of science and learned hands-on tasks in an ad hoc manner. As these staff became more senior they often trained the next generation of new and junior team members in in-house practices without reference to external common standards.

The publication of the International Society of Biological and Environmental Repositories (ISBER) Best Practices, National Cancer Institute (NCI) Best Practices for Biospecimen Resources, and the Organisation for Economic Cooperation and Development (OECD) guidelines for Human Biobanks and Genetic Research Databases were a very important first step toward sharing knowledge. These were widely accessed and the first two of these have become regularly updated "how-to" sources of biobank specific information. Annual ISBER and European, Middle Eastern and African Society for Biopreservation and Biobanking conferences included well attended in-person workshops on topics relevant to biobank staff and managers.
The next step was reflected by several local initiatives to develop and offer periodic topic-specific workshops or short courses, such as Biobanking and BioMolecular Resources Research Infrastructure "Hands on Biobanking" courses, some of which became the basis of more developed and formalized initiatives to create university masters and certificate level courses. This special section on education includes articles describing some of the biobank education programs now available from institutions in China, Europe, and North America. A companion "Experts Speaks" section also provides opinions from a range of biobanking experts on the most important topics to be emphasized in current and new biobanking courses.

The education programs described in this section are representative of a range of biobanking education programs that are now offered. The range can be considered in terms of types, primary delivery mechanisms, and learner groups. The three types of programs are master's programs, certificate courses, and workshops, which typically involve multiyear, multiweek, or single-/multiday commitments, respectively. Delivery mechanisms vary from a main emphasis on face-to-face methods to a main emphasis on online delivery. Learner groups include professional biobankers who need to learn about or update their knowledge of specific topics or scientists conducting some of the activities of biobanking, or nonbiobankers (e.g., ethics committee members, patient groups). Clearly each type of program and delivery mechanism offers different benefits and drawbacks in terms of access and time commitment as well as suitability, when considered from the perspective of the potential learner. This range of suitability reflects the diversity of backgrounds of learners from undergraduate and graduate students, to research and clinical laboratory technologists, to experienced biobank staff seeking to extend their knowledge.

The three examples described in this special section are intended to illustrate some of this diversity. All have been developed in the past 9 years and include the "University Biobank Certificate"' course offered by the Integrated Biobank of Luxembourg and the University of Luxembourg, the "Edx Research Biospecimen Methods" course, also a

\footnotetext{
${ }^{1}$ Plant and Crop Sciences Division, Nottingham Arabidopsis Stock Centre (NASC), University of Nottingham, Nottingham, United Kingdom.

${ }^{2}$ Université de Lyon, UMRS 449, Lyon, France.

${ }^{3}$ Laboratoire de Biologie Générale, Université Catholique de Lyon, Lyon, France.

${ }^{4}$ Reproduction et Développement Comparé, EPHE, Lyon, France.

${ }^{5}$ Department of Anatomy and Neurobiology, School of Basic Medical Sciences, Central South University, Changsha, China.

${ }^{6}$ Department of Pathology and Laboratory Medicine, Office of Biobank Education and Research, University of British Columbia, British Columbia, Canada.

${ }^{7}$ Biobanking and Biospecimen Research Services, Trev and Joyce Deeley Research Centre, BC Cancer, Victoria, Canada.
} 


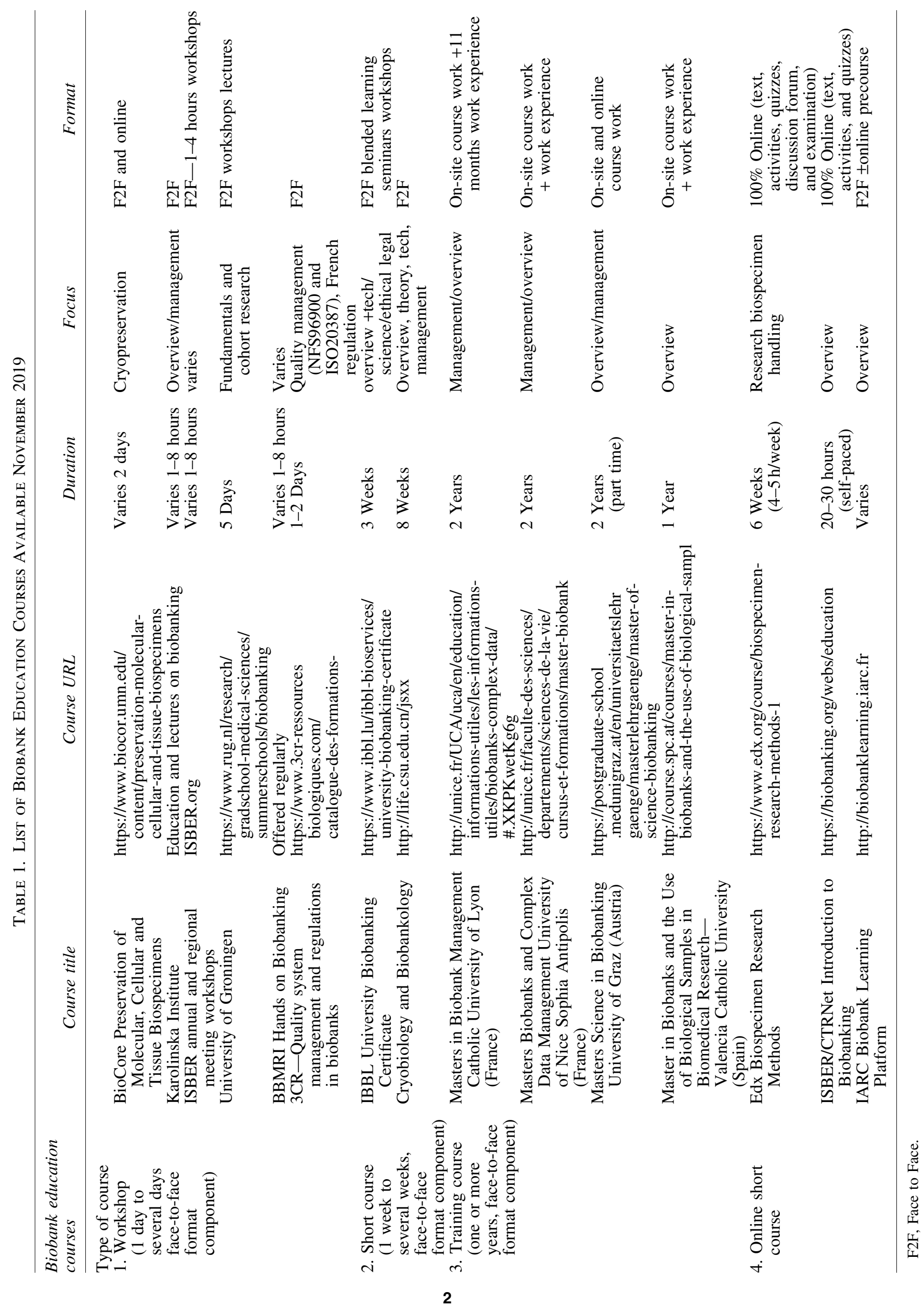


university certificate level course, offered by the University of British Columbia, Canada, and the graduate level "Cryobiology and Biobankology" course offered by the Central South University (CSU) in China. ${ }^{1-3}$ Other examples of masters level courses include the Masters Course in Biobank Management (University of Lyon, France), as previously described. ${ }^{4}$ See Table 1 for a current list of these and other biobanking education courses that is updated from the list Gormally et al. included in their 2017 article. ${ }^{4}$

All the courses described in these special section articles and previous articles cover the range of biobanking topics offered by each course, including number of learners who have matriculated, geographic origin of learners, and frequency of course offerings. A total of 395 learners have completed the four programs ${ }^{1-4}-665$ from the IBBL/ University of Luxembourg program, 68 from the University of Lyon program, 45 from the Central South University China program, and 217 have completed the University of British Columbia Edx program. These programs also report an international audience; for example, $38 \%$ of the countries of origin of students enrolled in the IBBL/University of Luxembourg are also from outside Europe, and 35\% of University of British Columbia Edx students are from outside North America. The CSU course is offered annually, the IBBL/University of Luxembourg course is offered every 2 years, the University of Lyon course is offered annually, and the University of British Columbia course is offered three times per year.

Although all three articles in the current special section ${ }^{1-3}$ report positive feedback from learners on multiple aspects of their respective courses, it is worth noting that the learners themselves often comment on the value of face-to-face learning in addition to online learning. This factor attests to the benefit of networking opportunities and hands-on experience, which is only possible with the face-to-face format. Entirely online courses, such as the UBC Edx course, can only partially address this by incorporating discussion boards and interactive sessions, but enable the widest geographic access as the trade-off.

\section{Conclusion}

Credentialing of biobank staff will become important as biobanking becomes professionalized, both within formal biobanks and also within research laboratories. Many edu- cation programs, such as those described in this special section and others listed in the table, now exist to support such credentialing.

However, it should be acknowledged that to date there is little published evidence/objective measurements of the impact of education programs on actual biospecimen and data quality, adherence to Standard Operating Procedures and standards, and so on.

Measuring the impact of education is challenging and so we need to work together to ensure that we critically evaluate our programs and share ideas to demonstrate their value to our donors, funders, universities, researchers, biobanks, and the public. In doing this we will be able to place higher value on the qualifications that are based on or arise from these courses and be able to provide organizations with evidence to establish and recognize biobanking as a more formalized career.

\section{Author Disclosure Statement}

No conflicting financial interests exist.

\section{References}

1. Castellanos-Uribe M, May ST, Betsou F. Integrated BioBank of Luxembourg-University of Luxembourg: University Biobanking Certificate. Biopreserv Biobank 2020.

2. Matzke L, O’Donoghue S, Watson PH. UBCx "Biospecimen Research Methods Course.” Biopreserv Biobank 2020.

3. Zhou H, Wu X, Zheng L, et al. The International Teaching and Practice of Cryobiology and Biobankology Course in China. Biopreserv Biobank 2020.

4. Gormally E, Hardy I, Caboux E, di Donato JH, Hofman P. Training the next generation of biobankers: A two-year master's course in the management of biobanks. Biopreserv Biobank 2017;15:438-450.

Address correspondence to: Peter H. Watson, MSc, MBBChir, FRCPC Biobanking and Biospecimen Research Services Trev and Joyce Deeley Research Centre $B C$ Cancer Victoria V8R 6V5 Canada

E-mail: pwatson@bccancer.bc.ca 